\title{
Deliberation in health-related headlines
}

Chenran Ning, Hongjun Wu, Yue Liu

\{cn257, hw434, y1992\}@ cornell.edu

\begin{abstract}
Just how big of a difference will deliberated thinking make in the digital age when judging whether the headline of a digital article is true or fake? Misinformation plagued the Chinese internet space, and fake news, especially related to health tips, often went viral on the internet with rapid speed. A previous study ${ }^{1}$ was previously conducted on political articles measuring the influence of partisanship on thinking deliberately. In this paper, we conducted a study on how deliberation influenced the accuracy of Chinese netizens distinguishing real and fake news headlines, using a similar experiment procedure from the above mentioned study. We found that deliberation reduces the possibility of these readers being misguided by fake health-related headlines. A similar trend of accuracy was observed when participants thought deliberately compared to the original study, despite using different topics on a different population of participants.
\end{abstract}

\section{Introduction}

\subsection{Main Research Question}

We live in a world where the recipients of information often go through a lot of information every day. Consequently, this leads to a vacuum of misinformation and so-called "fake news". The impact of such misinformation is not neglectable, for example, misinformation on a social media platform can lead to a mob storming the capital of the United States, attempting to overturn an election result. Therefore, it is crucial to study the ability of people to distinguish misinformation under certain circumstances.

This experiment builds on the works by B. Bago, et, al. In their paper "Fake news, fast and slow: Deliberation reduces belief in false (but not true) news headlines" 2 The authors discussed how people can fail to deliberate between true and false news. The key distinction lies in whether people use their intuition or not during their reasoning. In the original study, Bago quoted the main theory that he used to construct his study, which was derived from Evans \& Stanovich ${ }^{3}$ and Kahneman ${ }^{4}$. The theory states: "Intuition allows for quick automatic responses that are often based on heuristic cues, while effortful deliberation can override and correct intuitive responses." That is, by engaging

\footnotetext{
${ }^{1}$ Bago B, Rand DG, Pennycook G. Fake news, fast and slow: Deliberation reduces belief in false (but not true) news headlines. J Exp Psychol Gen. 2020 Aug;149(8):1608-1613. doi: 10.1037/xge0000729. Epub 2020 Jan 9. PMID: 31916834.

${ }^{2}$ Bago B, et al. Fake news, fast and slow: Deliberation reduces belief in false (but not true) news headlines..

${ }^{3}$ Evans, J. S. B., \& Stanovich, K. E. (2013). Dual-process theories of higher cognition advancing the debate. Perspectives on Psychological Science, 8(3), 223-241.

${ }^{4}$ Kahneman, D. (2011). Thinking, fast and slow. New York, NY: Farrar, Straus and Giroux.
} 
in reasoning, people can make more accurate beliefs. Our replication experiment also aims to testify to this theory.

Through this experiment, we can have a good understanding of what are the causes of people using their intuition to reason about information they receive (that is, System 1 thinking) rather than think critically with deliberation.

\subsection{Background}

On the Chinese internet, the majority of people use a mobile application called

"WeChat". WeChat combines functionality of online conversation, video conferencing, social media posting, mobile payment and micro-transaction, digital ID, COVID tracker code, and more. Needless to say, WeChat is one of the most essential applications in everyone's daily life in China.

With the platform's popularity increase comes risk. That is, when information is delivered to those who are not familiar with misinformation, fake news can be easily believed to be true. In an era where everyone cares about their own health more than any other period in history, fake health articles with a catchy headline can be especially appealing to many.

One of the biggest sources of such "micro articles" comes from independent publishers, called "Official Accounts" on WeChat. These articles are written by potentially unprofessional authors without peer review, similar to articles published on Twitter. Anyone can host their own "Official Account" with little to none investment.

For many people, especially the elderly, the "Official Accounts" are their primary source of information regarding health tips. Because people tend to follow accounts that share information that they agree with or with similar interest, it doesn't take much effort for someone to develop their own "Information Cocoon" and only absorb likely-minded information fed by biased official accounts, leading to beliefs in fake or overly exaggerated health tips or benefits. We chose to experiment on health because health tips are one of the easiest topics that can go viral on the internet, even if the information that it promotes is clearly fake.

Traditionally, with printed media, the reader would have adequate time to read an article and come up with their own conclusions. However, in the form of digital media, people spend less time thinking about the trustworthiness of information. Our study, therefore, focuses on if the time they indeed spend on reading is adequate enough for people to think with deliberation. 


\subsection{Key Experimental Treatment}

The goal of this experiment is to study the influence of time limits imposed on the participants and whether they will think with deliberation in limited time. This simulates

the cases where during people's day-to-day life, although unconsciously, they usually are willing to spend a small amount of time on a piece of information and quickly move on. Therefore, this replication experiment's setup remains consistent with the original research and we are controlling "time limit" as our target variable.

The setup is as follows: Participants are randomly and independently divided into two groups. Group 1 will receive one response baseline treatment where they are given news headlines and have unlimited time to decide if the health news headlines are fake or not. Group 2 will receive two treatments: initial response and final response, where they will have limited time judging the accuracy of the news headline and then unlimited time to judge all the headlines once again. We will elaborate more in Section 2.1.

\subsection{Key measure variables}

The independent variable will be the time available for judging the prompt (limited/unlimited). The dependent variable is the average accuracy rated for the news headlines and serves as our key metrics to measure participants' ability to discern misinformation. The rate of accuracy will indicate how much participants trust the health headlines.

\section{Experiment}

Our hypothesis is that deliberation facilitates accurate judgement of information people receive on their mobile devices.

\subsection{Experiment Procedure}

The first part for our experiment procedure is the collection of health-related news headlines. We did so by cherry picking news headlines from major and minor news outlets in China, as well as some well-known fake news sources. We collected 10 health-related news headlines from 10 different health-related topics to eliminate the possibility of familiarity to the topics. Among all of the questions, 5 are validated to be true and 5 are fake. We preserved the first paragraph of the news to provide a little more context and information participants could utilize them for their judgement.

This experiment is conducted through an online questionnaire. We presented selected participants from the population with two treatments and three versions of the questionnaire: "Untimed", "Stage 1", and "Stage 2". 
For Treatment 1, Group 1 participants received the "Untimed" version for the one response baseline result. For Treatment 2, participants in Group 2 receive "Stage 1" and "Stage 2" to record their initial response and final response. "Stage 2" and the "Untimed" version gives participants unlimited time to decide whether the health-related articles are trustworthy while Stage 1 gives participants only 10 seconds to make a judgement before the questionnaire proceeds to the next questions. To avoid cheating and submitting the answers quickly in unlimited questionnaires, participants will be assigned at least 20 seconds to deeply think about the headlines and skip is not allowed before 20 seconds. Unanswered questions will be filled in with a default value of 0.5 .

The authors conducted internal testing for different settings of the experiments, and decided that 10 seconds is a good amount of time for participants to read and give a preliminary judgement of the health-related news headlines but not sufficient for pondering or reading through the first paragraph of the news. Participants will judge by mainly using their System 1.

The order of the questions is shuffled for every participant. For each health-related news headline, participants will be given 5 choices and assign the headline a value of 0 to 1 in step of 0.25 indicating the trustworthiness of the headline ( 1 means very trustworthy).

\subsection{Recruiting Technique}

The online questionnaire is open for anyone to participate in our experiment. However, contrary to the original research, our targeted population is educated people who obtained or are pursuing a bachelor degree or above to see if the same phenomenon still applies. We distributed our questionnaire link on Wechat, Weibo, and BiliBili student groups in China.

All participants were given a brief introduction of our experiment. For limited tasks, we gave them 3 timed sample questions (unrelated to health) to practice in order to reduce the possibility of performance impairment caused by pressure. For unlimited tasks, we gave them 1 sample question to familiarize them with the given tasks.

\section{Results}

We post-processed the data by dropping the highest $20 \%$ and lowest $20 \%$ ratings ( $1 \mathrm{~s}$ and $0 \mathrm{~s}$ ) and found the truncated average and variance for both true and false headlines. We dropped these extreme values in order to eliminate the possibility that the participants might have prior knowledge to the headline related topics. Calculated scores demonstrate 
how much participants believe true or false headlines are valid. The scores and variances are shown in Table 1 below. And the final visualization of scores is shown in Figure 1.

\begin{tabular}{|l|l|l|l|l|}
\hline & true_score & false_score & true_var & false_var \\
\hline $\begin{array}{l}\text { Initial } \\
\text { response }\end{array}$ & 0.5096 & 0.6571 & 0.1212 & 0.1263 \\
\hline $\begin{array}{l}\text { Final } \\
\text { response }\end{array}$ & 0.6034 & 0.5769 & 0.1002 & 0.1129 \\
\hline $\begin{array}{l}\text { One response } \\
\text { baseline }\end{array}$ & 0.4167 & 0.4087 & 0.1034 & 0.0953 \\
\hline
\end{tabular}

Table 1: Experiment Results

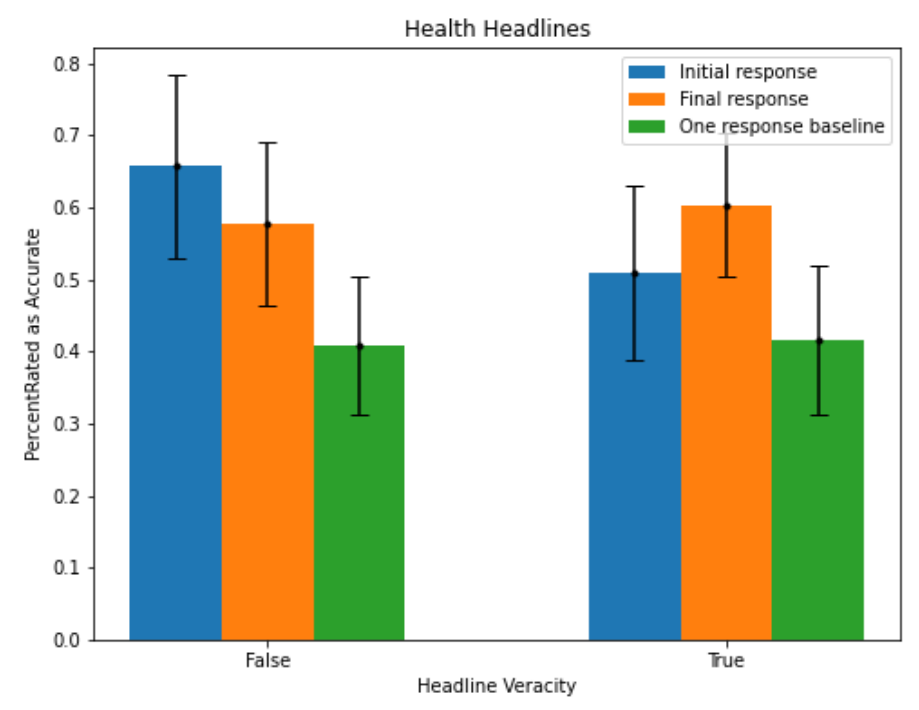

Figure 1: Percentage of true vs. false headlines that subjects rated as accurate across conditions

The X-axis in Fig. 1 represents the veracity of the health-related news headlines: whether the headline is fake or true. Y-axis represents the percentage of headlines that are categorized as accurate. For example, the last green bar represents that participants show an average of $40 \%$ trustiness towards genuine health-related news headlines.

\section{Discussion}

\subsection{Effects of deliberation}

In the hypothesis, when given more time and forcing participants to think with deliberation (participants have to wait for twenty seconds before allowing them to proceed to the next question in "Untimed" and "Stage 2"), we expect people would show 
higher accuracy when distinguishing the news headlines. In figure 1, the "True" blue bar is lower than the orange one. The accuracy of recognizing a news headline is "True" did increase after the forced think with deliberation, showing our participants are more likely to distinguish true news headlines if they use more time to think deliberately, and information about health naturally requires critical thinking to make sense and believe. Also, the "False" blue bar is higher than the orange one. The trust for headlines as "False" did decrease after deliberation, which is a good support to our hypothesis.

The initial response for false headlines has a higher percentage rated as accurate compared to initial response for true headlines. This shows that our target audiences are more likely to believe false headlines, and therefore, we can illustrate with statistics that fake news are more likely to go viral.

The variance remains consistent across all experiment and headline categories, this is what we expected as it represents the consistent discrepancy within participants in judging the trustworthiness of the health-related headlines.

\subsection{Insights from the "Untimed" one response baseline}

The accuracy of participants who received Treatment 1 shows an overall $\sim 2 \%$ accuracy, signifying that people usually have a neutral feeling towards news headlines. While the survey is untimed, it should be noted that Treatment 1 simulates the usual reading habits for ordinary readers. Even given more time, it is still possible that participants still read with their intuition due to day-to-day information digestion habits.

What is interesting is why the "one response baseline" has a lower accuracy $(\sim 40 \%)$ than the final responses $(\sim 59 \%)$. We believe that since participants in Group 2 are shown the same set of headlines twice, they become more familiar with these headlines and are more likely to believe them, whereas participants in Group 1 only see the headlines once. Because nowadays Chinese netizens are somewhat culturally against health-related headlines due to an overly populated flood of fake information, they are naturally more skeptical upon first sight.

\subsection{Data Comparison with Original Study}

The percentage of true vs. false headlines that subjects rated as accurate across conditions marked by participants is always around 50\% (Figure 1), which is significantly higher than the original research. The main factor should be that we set 5 choices rather than two choices with 0 and 1 . We set 5 choices because we cannot recruit enough participants to get large amounts of data for analysis to reach the standard of statistics. Thus, people may set their default choice to 0.5 . We also believe one of the factors that potentially caused this to happen is an education level that is higher than average. Another potential factor 
of influence might be due to the existence of a long history of misinformation in health-related news in China, causing people to feel skeptical towards health-related headlines which naturally may have contributed to the difference.

\subsection{Reflections on Ambiguous Results}

The final response for true and false headlines have approximately the same percentage rated as accurate. We believe that this particular value $(\sim 59 \%)$ is the population attitude towards all health-related headlines. However, it is possible to interpret such data as the participants aren't really distinguishing anything. This requires future research to explore the relationship between final response and one-response baseline towards health-related headlines and the average ratings of the population with larger groups of participants.

\section{Conclusion}

Deriving from the study we conducted above, deliberation does provide a visible understanding improvement to readers of digital articles who skim through headlines. Reading and thinking with deliberation reduces the possibility of these readers being misguided by only looking at the headline. However, it should be noted that the authors did not observe a dramatic change in the participants' ability to discern whether a piece of information is truth or hoax, unlike the original study. The difference between the nature of these two topics, that these two topics require different ways of thinking, is a suspected cause of this mild change. Nevertheless, our replication project is a success because we observed the same trend as the original work, despite the topic of choice being drastically different. 


\section{Reference}

1. Bago B, Rand DG, Pennycook G. Fake news, fast and slow: Deliberation reduces belief in false (but not true) news headlines. J Exp Psychol Gen. 2020 Aug;149(8):1608-1613. doi: 10.1037/xge0000729. Epub 2020 Jan 9. PMID: 31916834.

2. Evans, J. S. B., \& Stanovich, K. E. (2013). Dual-process theories of higher cognition advancing the debate. Perspectives on Psychological Science, 8(3), 223-241.

3. Kahneman, D. (2011). Thinking, fast and slow. New York, NY: Farrar, Straus and Giroux. 


\section{Appendix}

\section{A. Translation of Survey}

In order to better understand the experiment, a translated version of the original survey is needed, and we offer a sample of the original survey translated into English.

\begin{tabular}{|c|c|}
\hline Text in Chinese & Text in English \\
\hline $\begin{array}{c}\text { 冬天减肥效果更好? } \\
\text { 胜过跑步一小时的运动, 练起来, 快速瘦 } \\
\text { 高强度间歇训练 + 搏击, } \\
\text { 暴击脂肪, 快速减肥又减压 } \\
\text { 最好的养生方法, 医生说都是不用花钱 } \\
\text { 的! } \\
\text { This exercise is better than running for an } \\
\text { hour! Do it, and be fit quickly. } \\
\text { Intense training with interval resting + boxing, } \\
\text { tackles body fat and reduces anxiety }\end{array}$ \\
$\begin{array}{c}\text { The doctors always say that the best technique } \\
\text { to keep yourself healthy is free of charge! }\end{array}$ \\
Joy is the best health supplement!
\end{tabular}

Appendix A.1. Samples of true news headlines.

\begin{tabular}{|c|c|}
\hline Text in Chinese & Text in English \\
\hline $\begin{array}{c}\text { 吃酱油会致癌, 导致皮肤变黑 } \\
\text { 六招教你挑选好酱油 }\end{array}$ & $\begin{array}{l}\text { Soy sauce will actually cause cancer and make } \\
\text { skin go dark } \\
\text { Here are six tips for picking the best soy sauce }\end{array}$ \\
\hline $\begin{array}{l}\text { 坚持晚上用“醋”泡脚，会给身体带来哪些 } \\
\text { 变化呢？ }\end{array}$ & $\begin{array}{l}\text { What kind of miracles will keep foot bathing } \\
\text { with vinegar make to your body? }\end{array}$ \\
\hline 早知道早受益！ & $\begin{array}{l}\text { The earlier you know, the more benefit you } \\
\text { will get! }\end{array}$ \\
\hline
\end{tabular}

Appendix A.2. Samples of false news headlines. 